\title{
CARACTÉRISTIQUES COMMUNES DE L'INVASION DE LA CELLULE-HÔTE PAR LES SPOROZOAIRES
}

\author{
J. F. DUBREMETZ
}

\begin{abstract}
RÉSUMÉ
Les zoïtes de Sporozoaires possèdent des organites caractéristiques impliqués dans l'invasion de la cellule-hôte, un processus très conservé au sein du groupe. Les travaux réalisés sur différents genres ont montré d'étroites similitudes dans l'édification de la vacuole parasitophore, l'exocytose des organites et l'intégra-
\end{abstract}

tion de leur contenu dans la vacuole ou la membrane vacuolaire. Il est raisonnable de penser que la confrontation des résultats de l'étude de l'invasion dans les divers genres permettra de dépasser les particularités spécifiques et d'identifier les mécanismes fondamentaux du phénomène.
Summary : Commun features of host cell invasion by sporozoa.

Sporozoan zoites contain specific organelles that are involved in host cell invasion, a process highly conserved within this phylum. The data obtained on different genera have shown high similarities in parasitophorous vacuole development, organelle exocytosis and integration of the organelle contents into the vacuole or the vacuolar membrane. Parallel investigations on invasion process in different genera will help sorting fundamental mecanisms of Sporozoan invasion out of the specific peculiarities.
Bien que responsables de pathologies humaines ou animales aussi diverses que le paludisme, la toxoplasmose, la piroplasmose, la theileriose, la coccidiose ou la cryptosporidiose, tous les Sporozoaires partagent d'étroites similitudes structurales et biologiques dont l'une des principales est l'invasion de la cellule-hôte par les stades infectieux également appelés zoïtes. En effet, tous les stades infectieux des Sporozoaires, quelle que soit la complexité du cycle, possèdent un ensemble de différenciations caractéristiques regroupées sous l'appellation de «complexe apical ». Un nombre croissant de travaux relatifs à différents genres tendent à établir que les organites du complexe apical sont impliqués dans l'invasion de la cellule-hôte et jouent des rôles similaires dans un processus hautement conservé à travers le groupe.

\section{ÉTUDE MORPHOLOGIQUE DE L'INVASION}

La caractéristique majeure de l'invasion de la cellulehôte par les zoïtes de Sporozoaires est l'internalisation du parasite sans interruption du plasmalemme de la cellulehôte. Le parasite se retrouve ainsi dans un compartiment limité par une membrane et dénommé vacuole parasitophore.

La controverse qui oppose invasion active à phagocy-

Unité 42 INSERM, 369, rue J.-Guesde, F 59655 Villeneuved'Ascq Cedex, France. tose pour expliquer la pénétration des parasites tels Toxoplasma dans des phagocytes professionnels n'est pas close. De nombreux arguments plaident en faveur de l'invasion active d'autant que des cellules non phagocytaires peuvent être envahies, à moins d'admettre, dans ce cas, la coexistence de deux mécanismes.

Toutes les descriptions précises du processus d'invasion ont montré l'existence d'une jonction mobile au niveau de l'entrée du parasite dans la vacuole, quels que soient le zoïte et le type cellulaire envahi (Aikawa et al., 1978, 1981). Cette jonction qui est un contact étroit entre les plasmalemmes des partenaires se traduit, en cryofracture dans la membrane de la cellule-hôte, par une structure organisée identifiée initialement comme formée de particules intramembranaires mais qui pourrait plutôt correspondre à une réorganisation locale de la bicouche lipidique. En effet, une telle cristallisation pourrait expliquer la barrière opposée par cette jonction à la diffusion des protéines transmembranaires du plasmalemme-hôte vers la membrane vacuolaire en formation.

La déplétion quasi totale en particules intramembranaires de la membrane vacuolaire néoformée est également une caractéristique constante de l'invasion. La désorganisation du cytosquelette sous-jacent à la membrane de la cellulehôte serait une condition nécessaire au développement de la vacuole et des arguments plaident en faveur de cette hypothèse chez Plasmodium.

L'exocytose des rhoptries pendant le processus d'invasion a d'abord été postulée après l'observation d'organites 
vides pendant ou après le phénomène. De plus, il a quelquefois été possible d'observer des rhoptries ouvertes à l'apex du parasite selon une image d'exocytose typique. Plus récemment, l'immunolocalisation ultrastructurale de protéines provenant de ces organites dans la membrane vacuolaire a permis de confirmer cette exocytose. Le devenir des micronèmes est beaucoup moins clair et l'observation ultrastructurale n'a pas encore permis de suivre leur exocytose éventuelle.

\section{RECONNAISSANCE}

Cette étape du phénomène est sans doute celle qui présente le plus de variations au sein des Sporozoaires. Elle semble jouer un rôle crucial chez les parasites érythrocytaires pour lesquels de nombreuses études ont apporté quelques informations sur les mécanismes d'adhésion zoïtecellule-hôte préalables à l'internalisation (Mitchell et Bannister, 1988). Il se pourrait que des phénomènes de reconnaissance interviennent également pour des Sporozoaires capables d'envahir un très large éventail de cellules-hôtes in vitro et que ceux-ci pourraient moduler l'efficacité relative d'invasion des divers types cellulaires.

\section{PHYSIOLOGIE DE L'INVASION}

La mobilité des zoïtes de Sporozoaires s'opère par glissement, c'est-à-dire par translocation d'une interaction entre la surface du protozoaire et un support. Elle est orientée, la région apicale étant toujours antérieure. Le moteur moléculaire de cette motilité est inconnu mais il est tentant de postuler qu'il puisse être commun au glissement, au capping de ligands ou de particules et à l'invasion (King, 1988). La cytochalasine B inhibe tous ces processus chez les Sporozoaires, ce qui suggère que l'actine puisse être impliquée dans le glissement et donc dans l'invasion.

\section{CONTRIBUTION DES ORGANITES}

A L'ÉDIFICATION DE LA VACUOLE PARASITOPHORE

Les organites du complexe apical sont de trois types : rhoptries, micronèmes, granules denses. Ces derniers qui ont été longtemps négligés ou confondus avec des sections de rhoptries sont à présent l'objet d'une attention soutenue car il est de plus en plus évident qu'ils participent à la phase terminale de l'invasion, ou, plus tard, au début de la vie intracellulaire.

Les rhoptries ont été les organites plus étudiés jusqu'à présent. Dans les modèles les plus connus, les protéines de rhoptries ont constitué jusque-là l'essentiel des molécules identifiées dans ces organites. Une caractéristique intéressante concerne l'existence de familles de protéines por- tant des déterminants antigéniques communs (Sadak et al., 1988). Une autre est l'hétérogénéité du contenu : des protéines sont exclusivement localisées dans le pédoncule, alors que d'autres sont trouvées dans la partie postérieure renflée (Holder et al., 1985; Roger et al., 1988). Une telle richesse structurale et de contenu suggère une grande complexité fonctionnelle dont l'interprétation est encore hors de portée. La présence de phospholipides pouvant être à l'origine de l'expansion de la membrane vacuolaire n'a pas été établie; dans les cas où les rhoptries ont pu être isolées par fractionnement subcellulaire, leur densité ou l'analyse de leur contenu ont suggéré que les phospholipides n'étaient pas un composant majeur de ces organites (Dubremetz et al., 1989). Les propriétés des protéines de rhoptries sont encore très mal connues et les activités enzymatiques supposées pour elles (protéases, phospholipases) sont encore à établir et n'ont été suggérées que par l'activité d'inhibiteurs sur l'invasion dans des modèles particuliers (Saffer et al., 1988). Une autre propriété également postulée antérieurement a été retrouvée chez Toxoplasma où la mise en évidence d'une famille de protéine de rhoptries de $\mathrm{pHi}$ très basique $(\mathrm{pHi}>8)$ suggère une possible intervention dans la modification du plasmalemme de la cellule-hôte.

Le déterminisme de l'exocytose des rhoptries est inconnu. Cependant, des éléments connus aux zoïtes, et ayant probablement une relation avec cet événement, sont également connus : le site d'exocytose porte une rosette de particules intramembranaires analogue au site d'ancrage des extrusomes de certains protozoaires et, comme chez ces derniers, la calmoduline est détectée à proximité du site.

Les micronèmes ont été décrits dans plusieurs genres, mais demeurent énigmatiques et sont l'objet d'un grand nombre d'hypothèses difficiles à concilier sauf si cet organite réalise simultanément ou séquentiellement plusieurs fonctions distinctes. En effet, ils sont supposés contenir :

- des précurseurs de protéines de surface du zoïte (Fine et al., 1984),

- le RESA, déversé lors de l'invasion sous la membrane érythrocytaire (Brown et al., 1985), ou d'autres molécules retrouvées plus tard au niveau des knobs ou caveolaes (Torii et al., 1989).

Par ailleurs, les micronèmes sont les organites pour lesquels les variations en quantité sont les plus importantes entre les différents Sporozoaires, voire entre les zoïtes des différents stades d'une même espèce. C'est ainsi que se pose la question, lorsqu'ils sont très abondants (occupant parfois plus du tiers du volume cellulaire), d'un rôle exclusif lors de l'invasion, ou également d'une contribution ultérieure lors du développement intracellulaire puisqu'ils se résorbent dans les stades précoces de la schizogonie.

Les granules denses, aussi appelés microsphères chez $P$. knowlesi où leur exocytose dans la vacuole parasitophore a été décrite pour la première fois (Bannister et al., 1975), sont exocytés latéralement, sans qu'un site particu- 
lier ait pu être défini (Torii et al., 1989). L'exocytose intervient généralement très tôt après le début de la vie intravacuolaire. Chez Sarcocystis, l'événement est compliqué par la formation d'une vacuole parasitophore secondaire, non observée chez les autres Sporozoaires (Entzeroth et al., 1986). Chez les tachyzoïtes de Toxoplasma, l'exocytose des granules denses intervient après l'invasion (Sibley et al., 1988), mais semble également avoir lieu en phase extracellulaire (Cesbron et al., 1989). Le rôle de l'exocytose des granules denses dans la vacuole n'est pas établi, bien que les observations suggèrent que leur contenu s'associe à la membrane vacuolaire ou à des formations membranaires intravacuolaires. La mise en évidence d'un site de fixation du calcium sur une protéine des granules denses de Toxoplasma (Cesbron et al., 1989) suggère une participation de ces protéines aux régulations ioniques du compartiment vacuolaire, aspect important du parasitisme intracellulaire encore totalement ignoré.

\section{PERSPECTIVES}

La similarité de structure des différents compartiments des zoïtes de Sporozoaires suggère pour chacun d'entre eux une similarité de fonction à travers le groupe. Ainsi que nous l'avons décrit ci-dessus, cette suggestion est confirmée par les résultats obtenus lors de l'étude de l'invasion dans la mesure où, chaque fois qu'un événement nouveau est décrit dans un modèle, ce résultat est généralement observé chez les autres membres du groupe, au moins dans ses grandes lignes. Il est donc légitime de penser que l'étude de chaque modèle peut apporter une contribution importante à la compréhension du mécanisme général de l'invasion des cellules par les zoïtes des Sporozoaires. Ceci est d'autant plus important que la comparaison des différents genres permet de dépasser les particularités spécifiques et d'identifier les aspects fondamentaux du mécanisme d'invasion.

Remerciements. - A. Achbarou, D. Camus, R. Entzeroth, E. Ferreira, B. Fortier, F. Foussard, M. Leriche, O. Puijalon, A. SADAK ont largement contribué aux résultats non encore publiés et à l'élaboration des hypothèses rapportés dans cette communication.

\section{RÉFÉRENCES}

Aikawa M., Miller L. H., Johnson J., Rabbege J. : Erythrocyte entry by malarial parasites. A moving junction between erythrocyte and parasite. J. Cell Biol., 1978, 77, 72-82.
Aikawa M., Miller L. H., Rabbege J., Epstein N. : Freeze-fracture study of the erythrocyte membrane during malarial parasite invasion. J. Cell Biol., 1981, 92, 55-62.

Bannister L. H., Butcher G. A., Dennis E. D., Mitchell G. H. : Structure and invasive behaviour of Plasmodium knowlesi merozoites in vitro. Parasitology, 1975, 71, 483-491.

Brown G. V., Culvenor J. G., Grewther P. E., Bianco A. E., Coppel R. L., Saint R. B., Stahl H. D., Kemp D. J., Anders R. E. : Localization of the ring-infected erythrocyte surface antigen (RESA) of Plasmodium falciparum in merozoites and ring-infected erythrocytes. J. Exp. Med., 1985, 162, 774-779.

Cesbron-Delauw M. F., Guy B., Torpier G., Pierce R. J., Lenzen G., Cesbron J. Y., Charif H., Lepage P., Darcy F., Lecocq J. P., Capron A. : Molecular characterization of a 23-kilodalton major antigen secreted by Toxoplasma gondii. Proc. Natl. Acad. Sci. U. S. A., 1989, 86, 7537-7541.

Dubremetz J. F., Ferreira E., Dissous C. : Isolation an partial characterization of rhoptries and micronemes from Eimeria nieschulzi zoites (Sporozoa Coccidia). Parasitol. Res., 1989, 75, 449-454.

Entzeroth R., Dubremetz J. F., Hodick D., Ferreira E. : Immunoelectronmicroscopic demonstration of the exocytosis of dense granules contents into the secondary parasitophorous vacuole of Sarcocystis muris (Protozoa Apicomplexa). Eur. J. Cell Biol., 1986, 41, 182-188.

Fine E., Aikawa M., Cochrane A. H., Nussenzweig R. S. : Immuno-electron microscopic observations on Plasmodium knowlesi sporozoites: localization of protective antigen and its precursors. Am. J. Trop. Med. Hyg., 1984, 33, 220-226.

Holder A. A., Freeman R. R., Uni S., Aikawa M. : Isolation of a Plasmodium falciparum rhoptry protein. Mol. Biochem. Parasitol., 1985, 14, 293-303.

King C. A. : Cell motility of Sporozoan Protozoa. Parasitol. Today, 1988, 4, 315-319.

Mitchell G. H., Bannister L. H. : Malaria parasite invasion: interactions with the red cell membrane. Crc Critical Reviews Oncol. Hematol., 1988, 8, 225-310.

Roger N., Dubremetz J. F., Delplace P., Fortier B., Tronchin G., Vernes A. : Characterization of a 225 kilodalton rhoptry protein of Plasmodium falciparum. Mol. Biochem. Parasitol., 1988, 27, 135-141.

Sadak A., Taghy Z., Fortier B., Dubremetz J.-F. : Characterization of a family of rhoptry proteins of Toxoplasma gondii. Mol. Biochem. Parasitol., 1988, 29, 203-211.

Safer L. D., Long Krug S. A., Schwartzman J. D. : The role of phospholipase in host cell penetration by Toxoplasma gondii. Am. J. Trop. Med. Hyg., 1989, 40, 145-149.

Sibley L. D., Krahenbuhl J. L. : Modification of host cell phagosomes by Toxoplasma gondii involves redistribution of surface proteins and secretion of a $32 \mathrm{kDa}$ protein. Eur. J. Cell Biol., 1988, 47, 81-87.

Torii M., Adams J. H., Miller L. H., Aikawa M. : Release of merozoite dense granules during erythrocyte invasion by Plasmodium knowlesi. Infect. Immun., 1989, 57, 3230-3233.

Torii M., Matsumoto Y., Kamboj K. K., Maracic M., Guo S. Q., Nussenzweig R. S., Aikawa M., Cochrane A. H. : Association of microneme antigens of Plasmodium brasilianum merozoites with knobs and other parasite-induced structures in host erythrocytes. Infect. Immun., 1989, 57, 596-601. 